auf seiten einzelner Wissenschaftler, keinesfalls aber um normierte soziale Verbindlichkeiten bestimmter Wissenschaftlergruppen handelt, wird verhindert, daß auch die Ergebnisse dieser Gebiete ,nur eine regionale Verbindlichkeit haben". Kein Wissenschaftler würde sich auf die Ergebnisse einer Nachbardisziplin ,verlassen", wenn er annehmen müßte, daß dort nicht die allgemeinen Regeln umfassender wissenschaftlicher Kritik, sondern bestimmte inhaltliche Überzeugungen (z. B. solche über die Vorzüge bestimmter Experimentalregeln) soziale Verbindlichkeit besitzen.

\section{Literatur}

BEN-DAVID, J., 1973: Probleme einer soziologischen Theorie der Wissenschaft. Unveröff. Ms. (erscheint voraussichtlich demnächst in: Perspektiven der Wissenschaftsforschung - Eine Vorlesungsreihe, hersg. von P. Weingart. Frankfurt: Athenäum.)
CRANE, D., 1969: Social structure in a group of scientists: A test of the ,invisible college" hypothesis. ASR XXXIV, 335-52.

CRANE, D., 1972: Invisible colleges. Chicago \& London The University of Chicago Press.

GRIFFITH, B.C., \& N.C. MULLINS, 1972: Coherent social groups in scientific change. Science CLXXVII, $959 \mathrm{ff}$

LAKATOS, I., \& A. MUSGRAVE (Hersg.), 1970: Criticism and the growth of knowledge. Cambridge: Cambridge University Press.

MUSGRAVE, A., 1971: Kuhn's second thoughts. Brit. J. Phil. Sci. 22, 287-306.

POPPER, K. R., 1973: Objektive Erkenntnis. Ein evolutionärer Entwurf. Hamburg: Hoffmann und Campe. (Objective knowledge. An evolutionary approach, Oxford 1972, deutsch).

\section{ROLF KLIMA}

Universität Bielefeld, Fakultät für Soziologie 48 Bielefeld, Kurt-Schumacher-Straße 6

\title{
Ein handlungstheoretisches Konzept der scientific community.
} Skizze zu weiteren theoretischen Arbeiten - Antwort auf ROLF KLIMA

1. ROLF KLIMA hat in seinen vorstehenden kritischen Bemerkungen zu meinem Aufsatz über Experimentalregeln behauptet, daß der beabsichtigte Übergang von wissenschaftstheoretischen $\mathrm{zu}$ wissenschaftssoziologischen Kategorien nicht geleistet sei und auch nicht geleistet werden könne. Dabei hat er im Laufe unserer Auseinandersetzung seine Argumentation spezifiziert bzw. verschärft: Während er in einer ersten Fassung seiner Einwände verlangte, daß man ,genuin“ soziologische Begriffe wie Sanktion, Institution, Einfluß, soziale Kontrolle, Wettbewerb, Macht, Kommunikation erreiche, spezifiziert er sich in der vorliegenden auf den Zusammenhang von Norm und Sanktion: wenn die Einhaltung einer Norm nicht durch Sanktionen erzwungen wird, kann man den Geltungsbereich der Norm nicht als soziales Gebilde ansehen, sondern allenfalls davon sprechen, daß man sich durch Verletzung der Norm aus einem Tätigkeitsbereich herausdefiniert. Während er zunächst noch zwei Möglichkeiten, wissenschaftstheoretische und wissenschaftssoziologische Kategorien miteinander zu vermitteln, sah - nämlich einerseits das Studium quasi pathologischer Formationen in der Wissenschaft, in denen inhaltliche Uberzeugungen auto- ritativ erzwungen werden, andererseits das Studium epidemiologischer Formationen, bei denen sich über Netzwerke der Kommunikation wissenschaftliche Inhalte durch Abstimmung, Anpassung und Überredung verbreiten -, behauptet er nun, daß zumindest Untersuchungen der zweiten Art bereits die Irrelevanz inhaltlicher ,Verbindlichkeiten' für die soziale Formation gezeigt hätten.

Demgegenüber mußte ich feststellen, daß nicht als vorgegeben anzusehen ist, was es auf seiten der Soziologie zu erreichen gilt, weil nämlich meine Untersuchung eine Revision des soziologischen Begriffs der scientific community erzwingt. Diese Untersuchung bedarf deshalb nicht einer Ergänzung oder Korrektur, sondern vielmehr eines theoretischen backings, dessen Skizze unten folgt. Dabei handelt es sich nicht um den von KLIMA als modisch und bereits gängig unterstellten Versuch, die scientific communities durch einen gemeinsamen Besitz, oder besser: durch einen gegen Häresie sanktionierten Glauben zu bestimmen, sondern um eine Begriffsbildung von der informellen Organisation her, die sich durch Regeln wissen- 
schaftlicher Arbeit und Kommunikation ergibt. Der Ausgangspunkt meiner Überlegungen war die Feststellung, daß die bisherige Wissenschaftssoziologie zur Lösung bestimmter Aufgaben speziell der von KUHN gestellten, Wissenschaftsentwicklung als sozialen Prozeß zu verstehen nicht hinreichend gerüstet ist. Der Grund liegt wohl darin, daß sie scientific communities im wesentlichen vom reward-system oder faktischen Informationsflüssen her bestimmt. Nun setzt aber - um zum Beispiel mit STORER (The Social System of Science, 1966) zu sprechen das reward-system die Existenz von Fachgemeinschaften bereits voraus, denn nur aus ihrem Zirkel ist ,kompetente' Reaktion zu erwarten. Ebenso setzt der Ansatz der scientific community als Markt oder als Netz faktischer Kommunikation bereits voraus, daß ein von dem Zusammenhang wissenschaftlicher Arbeit her definierter Informationsbedarf besteht bzw. ein Argumentationszusammenhang, der Zitat und Verweis, Anknüpfung und Polemik sinnvoll macht. Daher meine Behauptung, daß sich Fachgemeinschaften von den Inhalten wissenschaftlicher Arbeit, von den spezifischen Regeln dieser Arbeit und vom Argumentationszusammenhang her konstituieren.

2. Mein eigener Ansatz geht nun davon aus, daß Wissenschaft selbst als ein Handeln zu verstehen ist. Wissenschaftliches Handeln läßt sich in drei Ebenen darstellen oder man sollte vielleicht besser sagen: in drei Sektionen. Die erste umfaßt die forschende Auseinandersetzung mit dem Gegenstand (Datenerzeugung, Experiment, Hypothesen- und Theorienbildung), die zweite die argumentative Auseinandersetzung zwischen den Wissenschaftlern und die dritte die Lehre, durch die die Regeln und Normen, die die erste und zweite Sektion beherrschen, tradiert werden. Soziale Formationen von Wissenschaftlern werden nun gebildet erstens dadurch, daß sie mit demselben Gegenstand zu tun haben: das wird gewährleistet durch die Institutionalisierung gewisser technisch-methodologischer Regeln; zweitens dadurch, daß sie miteinander in einem Argumentationszusammenhang stehen. Die patterns der Wissenschaftstradition, d.h. also die für die dritte Sektion maßgeblichen Regeln, sind nicht furr die Konstitution, sondern für die Reproduktion wissenschaftlicher Gemeinschaften verantwortlich. Die wissenschaftssoziologisch entscheidende These ist nun, daß das organisierende Prinzip wissenschaftlicher Gemeinschaften die Argu- mentation ist. Wissenschaftliche Gemeinschaften sind als Argumentationsgemeinschaften anzusehen, jede wissenschaftliche Publikation als ein Argument.

\section{Mit dem Begriff des ,organisierenden Prinzips“} möchte ich den Interaktionstyp herausheben, der eine soziale Formation zusammenhält, $\mathrm{zu}$ einem Ganzen macht und nach außen abgrenzt. Das organisierende Prinzip soll zugleich für die soziale Formation konstitutiv sein. Das heißt, daß der als organisierendes Prinzip zu bezeichnende Typ komplexer Interaktion die soziale Formation zu dem machen soll, was sie ist, ohne $\mathrm{da}$ dafür andere Interaktionstypen vorauszusetzen wären. Daß auch nicht-konstitutive Interaktionen zur Systembildung ausreichen können, hat STORER für den Austausch von kreativer Leistung und kompetenter Reaktion gezeigt.

\section{Nach PARSONS ist jede Handlung in dreier-} lei Weise orientiert, nämlich kognitiv, affektiv und evaluativ, oder sagen wir kognitiv, motivational und intentional. Will man nun die Wissenschaftssoziologie im Rahmen einer allgemeinen Handlungstheorie aufbauen, so ist als erstes zu beachten, daß wissenschaftliches Handeln primär kognitiv orientiert ist. Dies ist sicherlich eine Besonderheit des Wissenschaftssystems, in anderen Handlungssystemen spielt die kognitive Orientierung eher eine untergeordnete, nämlich instrumentale Rolle. Gerade aber diese Besonderheit nicht beachtet zu haben, ist der Mangel der bisherigen Wissenschaftssoziologie: sie hat das System wissenschaftlichen Handelns von der motivationalen Ebene her aufgebaut. Dies gilt vor allem für STORER, weniger für MERTON, für den eher die intentionale Ebene die primäre war. Aber gerade an STORERS Kritik an MERTON kann man erkennen, wie diese Bevorzugung der motivationalen Ebene zustande kam: es war die Absicht, eine Theorie aufzubauen, die nicht nur Wie-Fragen, sondern auch Warum-Fragen zu beantwortet gestattet, oder schärfer gesagt (indem man die Zweideutigkeit des Warum wegbringt), eine Theorie, die einem erlaubt, aus Ursachen zu erklären. Nun ist eine solche Theorie sicherlich nicht überflüssig und auch nicht falsch, aber doch unzulänglich, weil man vieles im Wissenschaftssystem nicht aus Ursachen erklären kann, sondern aus Gründen erklären muß.

Außer dem Primat der kognitiven Orientierung 
muß man für die Wissenschaftssoziologie die Objektorientierung wissenschaftlichen Handelns beachten. Was in anderen Interaktionen eher nur instrumentelle Bedeutung hat, nämlich the location and characterization of objects (PARSONS/SHILS), ist hier das eigentliche Thema. Als Grundschema wissenschaftlichen Handelns ist bei der Beschränkung auf zwei Personen deshalb ein Dreieck aus zwei Wissenschaftlern und einem Objekt anzusetzen. Die Wissenschaftler sind dabei indirekt miteinander durch die Identität ihres Objektes verbunden, direkt durch ihre Interaktion. Auf der kognitiven Ebene wird die Beziehung zum Objekt durch die Forschung (Datenerzeugung, Experiment, Theorienbildung usw.), die Beziehung zum anderen Wissenschaftler durch Argumentation gegeben: Auf der motivationalen Ebene ist die Beziehung zum Objekt durch Neugier und das Gefühl der Kreativität gegeben, die Beziehung zum anderen Wissenschaftler durch gegenseitige Anerkennung. kognitive Verwendung seiner Behauptung hat dann die affektive Bedeutung eines rewards für ihn, muß aber wegen des unpersönlichen Charakters des kognitiven Gehaltes durch bestimmte Normen, etwa Zitationspflicht, für ihn sichergestellt werden. Ist die Reaktion seines Kommunikationspartners kognitiv gesehen eine Widerlegung, so bedeutet diese für ihn affektiv eine Deprivation. Man sieht so, wie die Äußerung von bestimmten Argumenten im Argumentationszusammenhang als Behauptung, Widerlegung, Einwand, Stützung (corroboration) je ihre kathektische Bedeutung haben. Als was sie aber jeweils auftreten können, bestimmt sich aus dem Argumentationszusammenhang. Natürlich können nun auch Äußerungen der Anerkennung außerhalb von Argumentationen auftreten. Aber dann sind sie entweder keine kompetenten Reaktionen im Sinne von STORER, oder aber sie setzen einen Argumentationszusammenhang und die Durchführung von konkreten Argumentationen kognitive Ebene

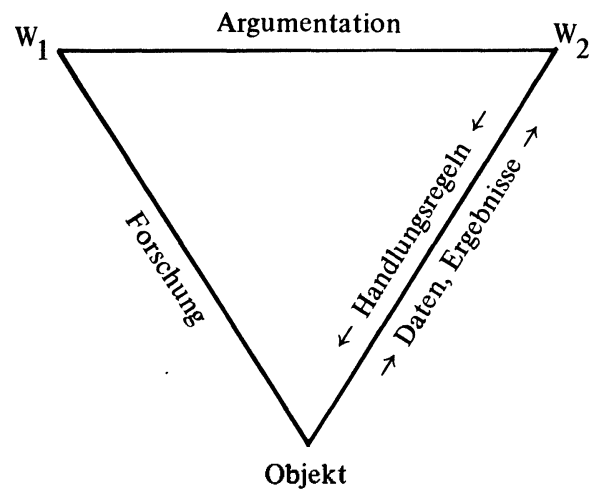

Die hier auseinandergelegten beiden Schichten wissenschaftlichen Handelns sind natürlich in jedem konkreten Handeln anzutreffen und nur analytisch als Momente trennbar. Um das kurz für die Argumentation klar zu machen: Wenn jemand etwas behauptet, so verbindet er mit der Mitteilung eines bestimmten kognitiven Gehaltes das Interesse, überhaupt Beachtung und ferner Zustimmung zu finden. Mit ersterem kann er nur rechnen, wenn er sich in einen bestimmten Argumentationszusammenhang hineinstellt. Letzteres würde etwa darin bestehen, daß ein anderer Wissenschaftler seine These übernimmt und daraus weitere Folgerungen zieht. Diese motivationale Ebene

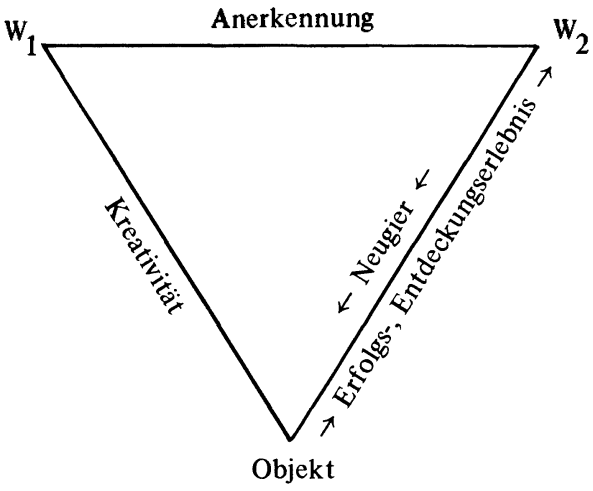

voraus und sind nur eine nachträgliche Abstraktion aus diesen.

Es ist nun offenbar möglich, daß Interaktionen sowohl auf der kognitiven als auch auf der motivationalen Ebene Anlaß zur Systembildung geben. Gerade dies ist bei sozialen Formationen in der Wissenschaft der Fall. Wir haben damit zu rechnen, daß die sich ergebenden Systeme durchaus nicht immer deckungsgleich sind und vor allem damit, daß es Spannungen zwischen ihnen gibt. Die grundlegende Systembildung, das organisierende Prinzip befindet sich aber stets auf der kognitiven Ebene. 
4. Da die Argumentation das organisierende Prinzip der scientific communities abgibt, ist ihre soziologische Bedeutung klar. Bisher ist aber noch nicht der für die Wissenschaft wesentliche Gegenstandsbezug jedes Handelns soziologisch interpretiert worden. Und hier liegt natürlich die Bedeutung von Experimentalregeln. Ich habe in dem Aufsatz gesagt, daß die Experimentalregeln für eine Fachgemeinschaft sichern, daß sie es mit demselben Gegenstand zu tun hat und daß sie damit zugleich die Abgrenzung gegen andere Fachgemeinschaften leisten.

Der phänomenologische Sinn dieser Aussage ist klar: die Membranforscher sind diejenigen, die sich mit der Zellmembran beschäftigen, die Entomologen diejenigen, die sich mit den Insekten beschäftigen usw. Nun ist natürlich allgemein soziologisch noch nicht einsichtig, warum der Gegenstand des Handelns irgendeine Bedeutung für die soziale Zusammenfassung der Akteure haben soll. Geläufig ist so etwas allenfalls furr den Fall, daß der Gegenstand das Ziel des kooperativen Handelns ist, etwa die Brücke für die an ihrem Bau beteiligten Personen und Firmen. Das Besondere am wissenschaftlichen Gegenstandsbezug ist aber, daß dieser im Unterschied zum gewöhnlichen, lebensweltlichen, nicht ein diffuser, sondern ein spezifischer ist: der Gegenstand dieses Handelns ist nicht schlechthin, sondern nur in einer bestimmten Zugangsart gegeben. Die Identität dieser Zugangsart muß aber über die Internalisierung bestimmter Handlungsregeln für jede wissenschaftliche Spezifität sichergestellt sein. Die Institutionalisierung und Internalisierung bestimmter Regeln des Verhaltens zum Objekt bringt nun natürlich die Wissenschaftler noch nicht in einen interaktionellen Zusammenhang, liefert jedoch eine wesentliche Voraussetzung dafür. Man könnte sagen, daß eine Fachgemeinschaft über Experimentalregeln und deren Sicherung des gemeinsamen Gegenstandes zum Kollektiv, aber noch nicht zum sozialen System wird. Letzteres ist erst mit der Etablierung eines Argumentationszusammenhanges gegeben. Die Experimentalregeln gehören also zu den Voraussetzungen des kognitiven Sozialsystems der Wissenschaft. Es wird durch sie eine gewisse Solidarität und ein Grundkonsensus sichergestellt. Dieser Grundkonsensus beruht auf praktischen, nicht sprachlichen Kompetenzen.

Priv.-Doz. Dr. GERNOT BÖHME

Max-Planck-Institut zur Erforschung der Lebensbedingungen der wiss.-techn. Welt

813 Starnberg, Riemerschmidstraße 7 